\title{
Web Services: Past, Present and Future
}

\author{
Sunny Kumar \\ Research Scholar \\ Swami Vivekanand University \\ Madhya Pradesh
}

\author{
Sanjeev Srivastava, PhD \\ Swami Vivekanand University \\ Madhya Pradesh
}

\author{
Amandeep Singh \\ Sri Sai lqbal College of \\ Management \& IT \\ Badhani, Pathankot
}

\begin{abstract}
In recent times, Web services have gained an amazing fascinates in both distributors as well as scientists. Web services permit accessibility to data that has formerly been locked within corporate and business systems and easily accessible only by using custom-made software .Web solutions, predicated on pre-existing Internet protocols and open guidelines, may offer an adaptable solution to the dilemma of application incorporation. With the support concerning WSDL, SOAP, and UDDI, Web services are growing to be prominent in Web applications. However, the current Web services architectures are challenged with just a few stubborn difficulties, as an instance, security. In this paper, we shall give an overview of these hassles. We feel that fixing these issues will end up imperative to success of Web services. The rest of the paper gives information about the three main technologies applied in association with Web Services: SOAP,WSDL, and UDDI.
\end{abstract}

\section{Keywords}

Web services, Security, Composition, Semantics, Grid services.

\section{INTRODUCTION}

In the previous couple of years, specially appointed methodologies have been utilized as a part of business-tobusiness applications to take positive aspect of the basic Internet infrastructure. Just Recently, Web services are growing being a methodical and extensible framework for application-to-application interaction, built on top of preexisting Web protocols and open XML standards.

Web services are a new reproduce associated with Web applications. They are self-contained, self-describing, modular methods which can be circulated, positioned, and invoked throughout the world wide web. Web services undertake functions that can be whatsoever from straight forward requirements for important information to making and executing challenging business procedures. Once a Web service is implemented, it could be determined and invoked by other applications (or other Web services).

The key feature of employing Web services is the ability to develop applications on the fly through the use of slackly coupled, reusable software elements. This has essential ramifications in both technologies and commercial applications. Software can be redelivered and remunerated for as fluid streams of services as in contrast towards prepackaged products and services. It's possible to accomplish automated as well as vibrant interoperability amongst systems to accomplish business responsibilities.

Business solutions could be entirely decentralized and dispersed over the web and used by way of a wide selection of communications appliances. Businesses can be published within the load of challenging, low and extravagant software incorporation and concentrate rather on the value of their attractions as well as objective important activities. Subsequently, the Internet will turned out to be a worldwide frequent platform where corporations and individuals communicate with almost every another to undertake assorted professional activities and to incorporate value added services. The hurdles in order to promoting new offerings and getting into emerging areas will likely be lessened make it possible access for small and medium-sized corporations. The enthusiastic enterprises and dynamic value chains become attainable and may also be even essential with regard to competitive advantages[5].

\section{TYPE OF WEB SERVICES}

Web Services tend to be programs that could be published, situated, as well as invoked throughout the Internet. Typical examples include

- Obtaining inventory cost suggestions

- Obtaining weather condition reports.

- Flight Reservations

These types of Web Services might use other Web Services in order to carry out their particular process. Up to now, there's no distinction between a Web Service and a server in a distributed application. The variations lie in the underlying levels for the carrying out the application logic and data adjustment. Regarding the Internet, generally there are heterogeneous surroundings on both the server and the client end, and it is not acknowledged beforeh and that which kind of middleware each end of the connection uses Mainly because Web Services represent just an additional perspective for the distributed applications, they are comprised of the identical three components. 
- A service agent which behaves as a look-up service in between a service provider and a service requestor.

- A service supplier that publishes their solutions to the service agent.

- A service requester that requests the service agent how to locate an appropriate vendor and therefore adheres itself to the service provider.

The subsequent design demonstrates the connections between the Web Services elements.

Fig 1:Web Service Components ${ }^{[9]}$

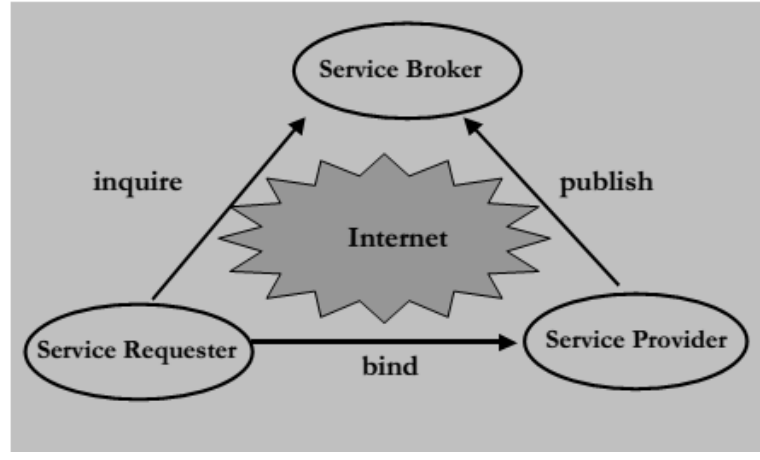

The Web services structure is split directly into three segments - communication protocols, service explanations, and service development. .

\section{Web Services: The PAST}

With the growth of computer networks, the perspective of dispersed computing ended up being produced. Applications happened to be split up initially straight into two section alongside one portion, the client, commencing a distributed exercise, and also the another a portion, the server, keeping away that movement. This particular decentralization lessened bottlenecks through dispersing the intensity all-around numerous components. It offered versatility towards application lay out earlier known as unidentified upon central hosts. But this particular two-tier design had actually its restrictions. For the failover and scalability, difficulties a third tier was brought out, isolating an application into a demonstration aspect, a middle tier formulated with the business logic, and a third tier interacting with all the data. This Specific three-tier unit of distribution has turned out to be typically the most popular technique for

dividing applications. It has a tendency in order to make application systems scalable. The groundwork when it comes to communication anywhere between the distributed section of an application is the remote procedure call (RPC). To always keep programmers through the low-level tasks such as data conversion or the byte order of a variety of devices, a unique layer of software strike the marketplace. This particular middleware masks the dissimilarities between numerous types of hosts. It is situated on top of the host's operating system and networking services and offers its services to the applications on top. The very first middlewares, such as DCE, had been according to a procedural programming model and were superseded by the launching of the object oriented programming model by middlewares like CORBA, ${ }^{\circledR}$ DCOM, or RMI, which have always been the widely accepted.

\section{Web Service: The Present}

As we have talked about, there are certainly essentially a variety of middlewares one can select from: DCOM, CORBA, as well as RMI. The whole bunch have their positive aspects along with their negative aspects. This particular section talks about the primary features without worry ing about too much into detail. It will factor out just how Web Services blend in to the envision.

\subsection{CORBA}

CORBA is an open standards-based remedy in order to distributed computing. The Object Management Group an industry syndicate, collectively the requirements for the CORBA as well as particular the world-wide-web Inter ORB Protocol[8], the traditional communication protocol between Object Request Brokers. The biggest benefit of CORBA is the fact that clients and servers can be written as part of any other programming lan guage. This Specific is quite possible mainly because objects are characterized by having a advanced level of abstraction offered by way of the Interface Definition Language.

\subsection{RMI}

Remote Method Invocation (RMI) makes it possible for you to definitely come up with distributed Java ${ }^{\mathrm{TM}}$-to-Java applications, in which their approaches to remote Java objects can be invoked from other Java virtual machines, potentially upon a variety of hosts. A Java program can easily produce a call on a remote object the moment it receives a reference point to the remote object, either by browsing within the remote object in the bootstrap naming service offered simply by RMI or by obtaining the reference point as being an argument or a return value. A individual can easily call a remote object in a server. Just That server can possibly be also a client of some other remote objects. RMI makes use of object serialization to marshal and unmarshal boundaries and will not truncate types, supporting true object-oriented polymorphism. The protocol utilized when it comes to communication is the Java Remote Method Protocol.

\subsection{DCOM}

Distributed Component Object Model (DCOM) makes it possible for calls to remote objects by means of working together with a layer that sits on foremost of their DCE RPC process and which one communicates with all the COM runtime services. A DCOM server publishes its techniques 
towards the consumers through promoting a number of user interface. These are generally written in IDL, which one is comparable to $\mathrm{C}++$. The IDL Compiler having proxies/stubs and skeletons similar to a CORBA IDL compiler, but registers themselves furthermore within the system's registry. In extension, RMI reinforcement dispersed garbage assortment.

Then again, you can just utilize it with Java on both sides of the association. What's more, the usability is attained to by keeping the middleware genuinely straightforward. It doesn't give any administrations as those predetermined by CORBA.

The paired level determination of DCOM permits the utilization of different dialects to code the server objects. Like RMI, DCOM backings appropriated junk accumulation of remote server objects

\section{Web Service: The Future}

Within article, we look at the subsequent specific features which are at the moment most outstanding and balanced in each subject.

1. The simple object access protocol (SOAP) that allows communications among the Web services.

2. The Web Services Description Language (WSDL) that provides a conventional, computer-readable explanation of Web services.

3. The Universal Description, Discovery and Integration (UDDI) directory that is a registry of Web services descriptions.

4. XML (Extensible Markup Language) is a text-based structure accustomed share information on the World Wide Web, intranets, and elsewhere.[7]

Fig 2 :Web Service Layer ${ }^{[9]}$

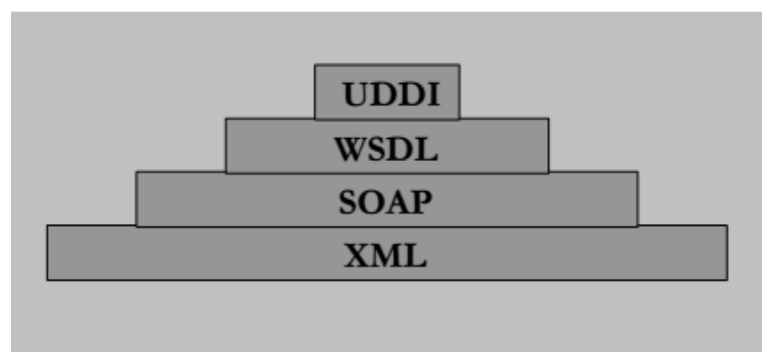

\subsection{SOAP}

SOAP is essentially a stateless, one-way communication exchange paradigm that allows applications to create more advanced relationship patterns (e.g., request/response, request/multiple responses, etc.) by incorporating one-way exchanges with characteristics offered by an underlying protocol and/or application-specific facts.

SOAP does not by itself identify any other application semantics[1] such as a programming model or implementation focused semantics, for example, distributed garbage set. It rather characterizes a simple process for showing application semantics by delivering a standard product packaging model and encoding mechanisms for encoding information within modules.

Even Though SOAP offers an excellent framework for information exchange, it is lacking in semantics regarding the application-specific information it conveys, such as the routing of SOAP messages, trustworthy information exchange, firewall traversal, etc. Also, SOAP offers a complete definition of the necessary steps taken by a SOAP node on obtaining a SOAP message ${ }^{[1]}$.

At its core, a SOAP message has a very straight forward framework: an XML element with two children elements, one that contains the header and the another the body. The header items and the entire body elements are also exemplified in XML.SOAP messages can be transferred over HTTP for the runtime invocation. The HTTP communications protocol perform the linking function for the fundamental interaction in between computer networks

\subsection{WSDL}

WSDL offers a model and an XML formatting for outlining Web services $\left.{ }^{[2}\right]$. WSDL characterizes services as collections of network endpoints or ports. In WSDL, the theoretical meaning of endpoints and messages is separated from their tangible network deploy ments or data structure bindings. This permits the reuse of conceptual meanings of messages that illustrate the data being exchanged and port types that represent collections of operations. The tangible protocol and data format requirements for a specific port type constitute a binding. A port is characterized by linking a network address with a binding. A range of ports defines a service.

WSDL identifies network services by making use of an XML $\operatorname{grammar}^{[2]}$. It offers documentation for distributed systems and has the goal to make it possible for applications to communicate with each other in an computerized way

\subsection{UDDI}

UDDI offers a mechanism for people to discover Web services ${ }^{[3]}$. Universal Description, Discovery, and Integration (UDDI) is a classique which is designed to offer a searchable directory of businesses and their Web Services. Thus, it signifies the service broker that makes it possible for service requesters to find a appropriate service provider. In numerous ways UDDI is manufactured such as a phone book.

Web services are meaningful only if potential clients may discover data sufficient to allow their execution. The emphasis of Universal Description Discovery \& Integration (UDDI) is the classification of a set of services supporting the description and development of (1) businesses, organizations, and other Web services providers, (2) the Web services they make accessible, and (3) the technological user interface 
which have been used to access those services. Based on a frequent group of enterprise expectations, including HTTP, XML, XML Schema, and SOAP, UDDI offers an interoperable, foundational framework for a Web servicesbased software ecosystem for both widely available services and services only subjected internally within an organization.

A UDDI registry is equivalent to a CORBA trader and can be regarded as as being a DNS service for business solutions. A UDDI registry has two varieties of clients: businesses who really want to publish a service classification (as well as its usage interfaces) and clients who would like to acquire services descriptions of a certain form and bind the services programmatically (using SOAP) ${ }^{[6]}$.

The UDDI data contains four levels. The top level is the business element that gives the general information about an organization, for example, its address, a short interpretation, contact data and other general identifiers. This kind of information can be observed due to the fact white pages of UDDI. Connected with each business entity is a checklist of business services, including the brief description of each and every service and the categories of the service, for instance, purchasing, shipping, etc. This can be considered as the yellow pages of UDDI. Within a business service, one or more binding themes determine the green pages that incorporate much more techie information regarding a Web service [4].

\subsection{XML}

The whole Web service approach is dependent on XML. Which pleads practical question, what exactly is XML? XML is an eXtensible Mark-up Language that makes it possible for you to determine as well as organize your important information within the considerably precise and versatile way. It is known as extensible mainly because it doesn't have a restricted format. An illustration of the mark up language which possesses predetermined structure is actually HYPERTEXT M ARKUP LANGUAGE, which is certainly an SML or Single Markup Language. The distinction amongst the two is that with XML you can determine any other component as well as call it whatever you like, while with HTML you absolutely come with pre-defined components as well as attributes. For example, in order to make text appear italic in a Web browser, you use the $\langle$ em $\rangle</$ em $\rangle$ tags. Solitary modern-day web browser may already be aware how to highlight that textual content. Without worry ing about predefined tags like this, XML enables you to outline your own personal tags to both display and format text. This Particular versatility is precisely what Web services make use of to really make it both platform and execution unbiased. XML is actually most popular in RSS documents, which tend to be useful for news distribution over the internet.

\section{CONCLUSION}

Having characterized one of the keys technologies, we right now introduce some sort of accomplish image of distributed applications as it is applicable to SOAP, WSDL, and UDDI:T

1.The Web Service provider represent the Web Service in a WSDL report as well as publishes it to a UDDI registration using the Publisher's API (that is dependent on SOAP).

2. A service requester makes use of the UDDI Enquiry API to look the UDDI registration for a appropriate vendor. If an individual is available, it would possibly seek out the Model directing to the WSDL document.

3. A SOAP request will be put together in accordance with the WSDL report.

4. The SOAP request will certainly be delivered to the service supplier, as well as their responses will likely be highly processed.

Although generating all of the essential documentation would seem complicated, please be aware that they're customarily produced comparable to stubs and skeletons in some other middlewares. The programmer does not have to worry in regards to the communication difficulties. Toolkits as well as APIs will certainly come up with conveniently the best XML documents.

All through this paper, Web Services were seen as "yet another middleware," like other middleware, for example, CORBA and RMI, yet doubtlessly more suitable to be utilized over the Internet. Thus, standard difficulties with regards to distributed application development such as overall performance, scalability, failover as well as protection need to be experienced additionally whenever handling Web Services. Just envision in case your own Web Service makes use of another Web Service for credit card verification. What will happen towards your Web Service or still towards your entire business if the security solution isn't available to you?Or exactly how should really your Web Service respond if it encounters a type of rush-hour alongside a huge number of client requirements? These problems will be the upcoming steps Web Services have actually to take in order to turned out to be a mature framework. 


\section{REFERENCES}

[1] SOAP Version 1.2 Part 0: Primer. Basic Usage Scenarios. http://www.w3.org/TR/2003/REC-soap 12part0-20030624/\#L1161.

[2] Web Services Description Language (WSDL) Version 1.2 Part 1: Core Language: http://www.w3.org/TR/2003/WD-wsdl12-20030611/.

[3] UDDI Version 3.0. UDDI Spec Technical Committee Specification, 19 July 2002 http://uddi.org/pubs/uddiv3.00-published-20020719.htm.

[4] Francisco Curbera, Matthew Duftler, Rania Khalaf, William Nagy, Nirmal Mukhi, and Sanjiva Weerawarana, Unraveling the Web Services Web, IEEE Internet Computing, March/April (2002) 86-93..

[5] D.Fensel, C.Bussle, Web Services Modeling Framework, Electronic Commerce Applications, 1(2002)113-137
[6] Francisco Curbera, Matthew Duftler, Rania Khalaf, William Nagy, Nirmal Mukhi, and Sanjiva Weerawarana, Unraveling the Web Services Web, IEEE Internet Computing, March/April (2002) 86-93

[7] V. Richard Benjamins, Web Services Solve Problems, and Problem-Solving Methods Provide Services, IEEE Intelligent Sy stems, January/February (2003) 76-77

[8] Christoph Bussler, Alexander Maedche, Dieter Fensel, Web Services: Quo Vadis? IEEE Intelligent Sy stems, January/February (2003)80-82

[9] Hartwig Gunzer, Sales En gineer, Borland,Introduction to Web Services

[10] Stefan Decker, Sergey Melnik, Frank Van Harmelen, Dieter Fensel, Michel Klein, Jeen Broekstra, Michael Erdmann and Ian Horrocks, The Semantic Web: The Roles of XML and RDF, IEEE Internet Computing, September • October 2000,63-74 